\title{
Tracking Single Molecule Dynamics in the Adult Drosophila Brain
}

\author{
(ㄱ)Adam D. Hines and ${ }^{\circledR}$ Bruno van Swinderen
}

https://doi.org/10.1523/ENEURO.0057-21.2021

Queensland Brain Institute, The University of Queensland, 4072 Brisbane, Queensland, Australia

\section{Visual Abstract}

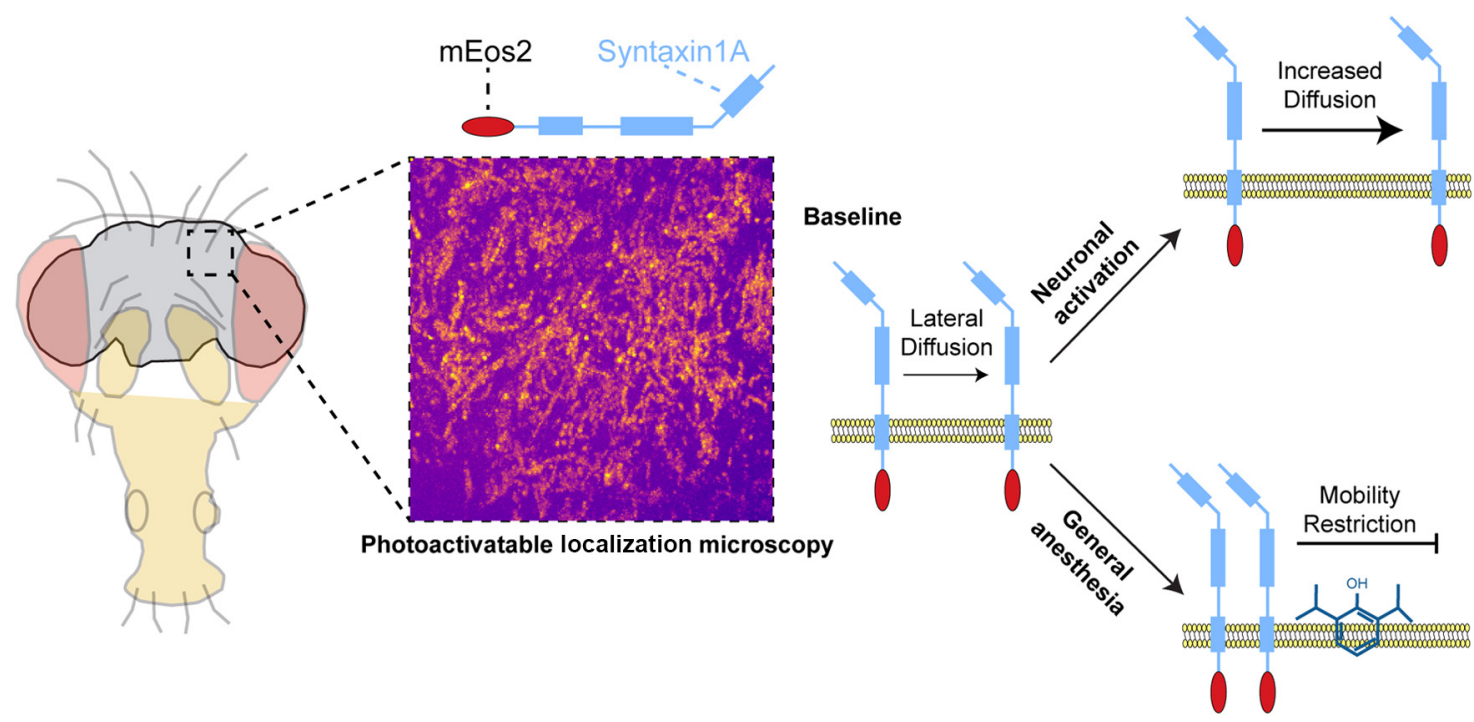

Super-resolution microscopy provides valuable insight for understanding the nanoscale organization within living tissue, although this method is typically restricted to cultured or dissociated cells. Here, we develop a method to track the mobility of individual proteins in ex vivo adult Drosophila melanogaster brains, focusing on a key component of the presynaptic release machinery, syntaxin1A (Sx1a). We show that individual Sx1a dynamics can be reliably tracked within neurons in the whole fly brain, and that the mobility of Sx1a molecules increases following conditional neural stimulation. We then apply this preparation to the problem of general anesthesia, to address how different anesthetics might affect single molecule dynamics in intact brain synapses. We find that propofol, etomidate, and isoflurane significantly impair Sx1a mobility, while ketamine and sevoflurane have little effect. Resolving single molecule dynamics in intact fly brains provides a novel approach to link localized molecular effects with systems-level phenomena such as general anesthesia.

Key words: brain; Drosophila melanogaster; ex vivo; general anesthesia; propofol; super-resolution microscopy

\section{Significance Statement}

Tracking the mobility of individual syntaxin1A (Sx1a) molecules in extracted fly brains provides a physiologically-relevant context for understanding the effects of neural activation and inhibition on protein dynamics in central neurons. 


\section{Introduction}

The brain of the fruit fly Drosophila melanogaster offers a rich platform to explore synaptic function at multiple levels, from detailed understanding of circuits to precise molecular mechanisms of chemical neurotransmission. A key advancement aiding our understanding of neurotransmission is the development of super-resolution microscopy, which allows for the visualization of proteins and molecules below the diffraction limit of light (Betzig et al., 2006; Willig et al., 2006). Super-resolution microscopy has provided novel insight on the nanoscale structure and dynamics of key components of the presynaptic release machinery, such as syntaxin1A (Sx1a; Ullrich et al., 2015; Bademosi et al., 2016; Reddy-Alla et al., 2017). Photoactivatable localization microscopy (PALM; Betzig et al., 2006) with single particle tracking (SPT) in live cells (Manley et al., 2008) allows molecules to be detected and followed through time in a variety of systems to explore macromolecular protein dynamics (Manzo and GarciaParajo, 2015). This has been made possible by the development of photoconvertible fluorophores such as Eos (McKinney et al., 2009; Zhang et al., 2012), which can be attached to proteins of interest to stochastically localize molecules sparsely and thereby study protein nanoscale organization, mobility, and diffusion in cells. To study Eos-tagged proteins, dual color illumination in a total internal reflection (TIRF; Axelrod, 2001) or highly inclined and laminated optical (HILO; Tokunaga et al., 2008) sheet configuration is employed to simultaneously record and stochastically photoconvert Eos fluorophores in cultured cells or dissociated neurons (Manzo and Garcia-Parajo, 2015). However, there is comparatively little information on single molecule dynamics in more complex living tissue, such as animal brains.

Recent studies have highlighted the value of performing super-resolution microscopy and sptPALM in intact tissue, revealing, for example, developmental changes that embryos undergo by imaging single molecule dynamics in their native environment (Chen et al., 2014; Mir et al., 2018; Reisser et al., 2018; Tønnesen et al., 2018). The importance of imaging in intact, native tissue was also

Received February 8, 2021; accepted March 26, 2021; First published April 19, 2021.

The authors declare no competing financial interests.

Author contributions: A.D.H. and B.v.S. designed research; A.D.H. performed research; B.v.S. contributed unpublished reagents/analytic tools; A.D.H. analyzed data; A.D.H. and B.v.S. wrote the paper.

This study was supported by National Health and Medical Research CouncilGrants GNT1065715 and GNT1164879 (to B.v.S.).The Zeiss ELYRA microscope was supported by the Australian Research Council Linkage Infrastructure, Equipment and Facilities (ARC LIEF)Grant LE130100078.

Acknowledgements: We thank Adekunle Bademosi and Merja Joensuu for critical discussions about the work, Rumelo Amor for help with microscopy, and the van Swinderen lab for feedback on the project.

Correspondence should be addressed to Bruno van Swinderen at b.vanswinderen@uq.edu.au.

https://doi.org/10.1523/ENEURO.0057-21.2021 Copyright (C) 2021 Hines et al.

This is an open-access article distributed under the terms of the Creative Commons Attribution 4.0 International license, which permits unrestricted use, distribution and reproduction in any medium provided that the original work is properly attributed. recently highlighted by uncovering unexpected results regarding the distribution of docked synaptic vesicles in Drosophila tissue compared with cultured mammalian chromaffin cells (Couteaux and Pecot-Dechavassine, 1970; Stevens et al., 2011; Jung et al., 2018). Here, synaptic vesicles in the fly larval neuromuscular junction are more readily docked and primed compared with chromaffin cells, suggesting important differences in the physiological relevance of the two systems for studying neurotransmission. We recently described single molecule imaging in intact motor nerve terminals of filleted Drosophila larvae (Bademosi et al., 2016, 2018a). In that study we tagged the presynaptic protein Sx1a with photoconvertible mEos2 and found that genetic stimulation of motoneurons resulted in increased mobility of Sx1A in the motor nerve terminals, suggesting increased mobilization of the presynaptic machinery when neurons are activated. In contrast, stimulation of chromaffin cells results in decreased Sx1a mobility (Kasula et al., 2016), highlighting that even highly conserved molecular mechanisms can differ depending on tissue type.

Sx1a is necessary for the docking and fusion of neurotransmitter-containing vesicles, and is a component of the SNARE complex along with its binding partners SNAP25 and VAMP2 (Südhof, 2012). Sx1a function is highly conserved in all animals (Bennett et al., 1992; Ferro-Novick and Jahn, 1994; Südhof and Rizo, 2011), with mutations in the protein often implicated in synaptic communication defects and lethality (Schulze et al., 1995; Saifee et al., 1998; Fergestad et al., 2001; Fujiwara et al., 2006; Vardar et al., 2016; Kofuji et al., 2017). Our growing understanding of the mechanisms underlying synaptic function has uncovered novel hypotheses for how neurotransmission might be compromised by certain drugs, such as general anesthetics (Hemmings et al., 2005, 2019; Humphrey et al., 2007; van Swinderen and Kottler, 2014; Baumgart et al., 2015; Bademosi et al., 2018b; Troup et al., 2019; Karunanithi et al., 2020). A Sx1a gain-of-function mutation was found to confer resistance to volatile general anesthetics in the nematode Caenorhabditis elegans (van Swinderen et al., 1999) as well as Drosophila flies (Troup et al., 2019), suggesting a potential presynaptic target mechanism for these drugs. Single molecule imaging of mEos-tagged Sx1a in Drosophila motor nerve terminals exposed to the sedative drug propofol revealed that this common general anesthetic may be immobilizing Sx1a into nanoclusters (Bademosi et al., 2018b). Thus, motor neuron activation and propofol exposure appeared to have opposite effects on Sx1a mobility in intact synapses, although these experiments were restricted to relatively large motor nerve terminals, so the relevance to smaller synapses in the brain remains unknown.

Here, we adapt super-resolution imaging and SPT techniques to the extracted adult fly brain and use this approach to determine whether Sx1a mobility can be acutely modulated in central synapses. Along with employing a thermogenetic neural activation paradigm, we test a panel of intravenous and volatile general anesthetics for potential effects on Sx1a mobility. We find that, 


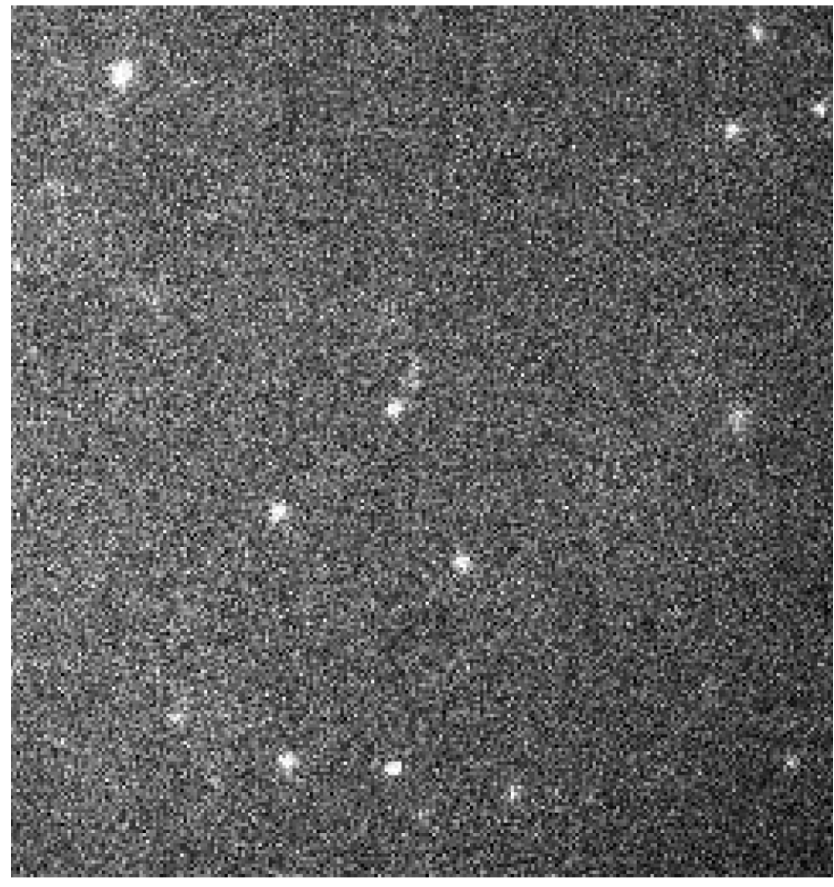

Movie 1. Tracking individual Sx1a-mEos2 molecules in the fly brain. [View online]

similar to Drosophila larval neuromuscular junction (Bademosi et al., 2016), the mobility of Sx1a molecules in the adult brain is increased on neuronal stimulation, thereby providing a physiologically relevant setting to probe for general anesthetic effects in intact brain tissue.

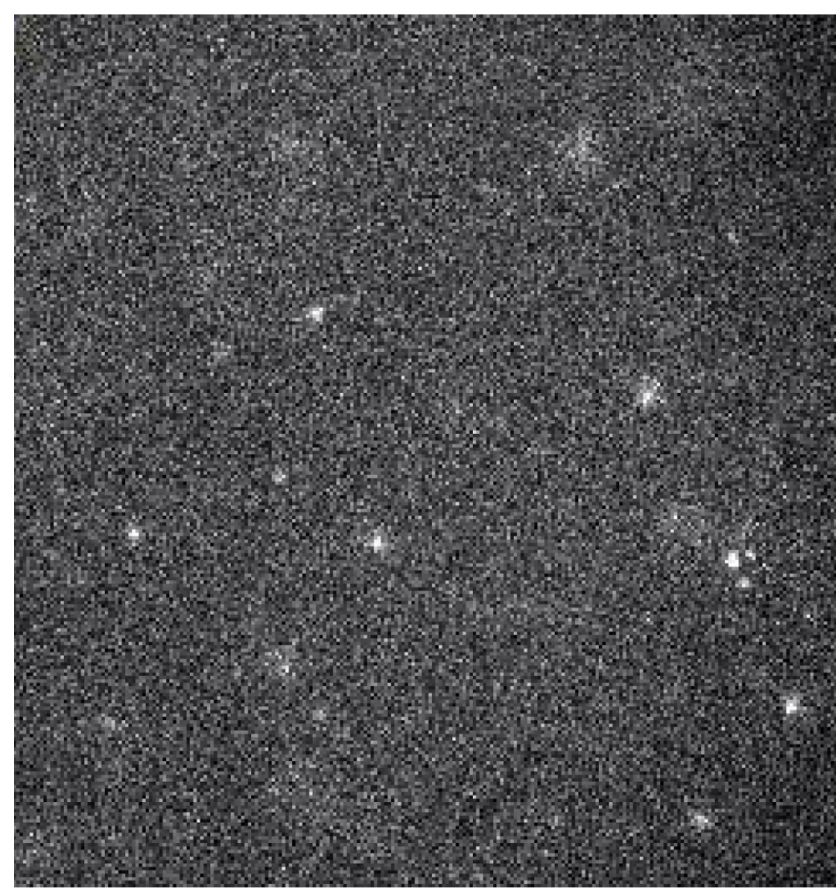

Movie 2. Absence of Sx1a-mEos2 mobility in fixed tissue. [View online]

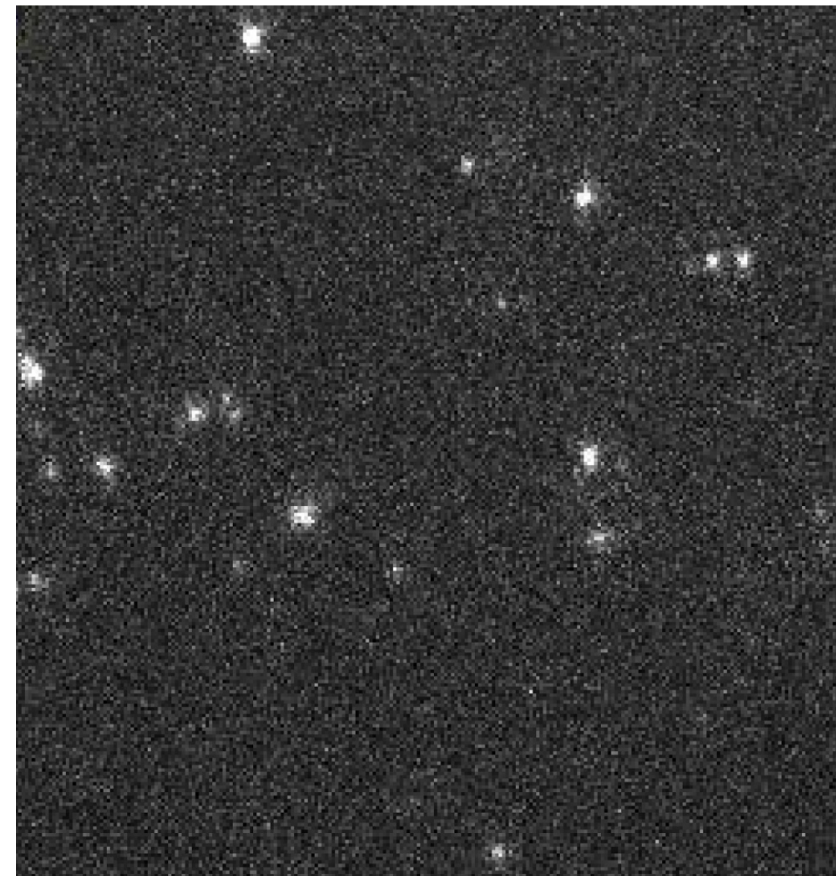

Movie 3. Absence of Sx1A-mEos2 tracking in HL3.1 solution. [View online]

\section{Materials and Methods}

\section{Fly stocks and rearing conditions}

Sx1a-mEos2 transgenic fly lines were generated as previously described (Bademosi et al., 2018a). Briefly, Sx1a cDNA was cloned to include a mEos2 tag by replacing the stop codon of Sx1a with a linker molecule GAGGTACCG CGGGCCCGGGATCCACCG. Whether mEos2 is appropriate for a $\mathrm{C}$-terminal or $\mathrm{N}$-terminal attachment depends on the protein of interest to study. Sx1a-mEos2 flies were injected with phiC31 onto the second chromosome and balanced with curly (Cyo). For dTrpA1 (Drosophila transient receptor potential cation channel 1a) experiments, $w^{1118}$; Sx1a-mEos2/Cyo;+/+ flies were crossed to a $w^{1118} ;+/+$; UAS-dTrpA1 line to generate a stable breeding stock with the genotype $\mathrm{w}^{1118}$;Sx1a-mEos2/Cyo;UAS-dTrpA1.

$D$. melanogaster fruit flies were reared on standard yeast-sugar-agar food in vials at $22^{\circ} \mathrm{C}$ with a $12 / 12 \mathrm{~h}$ light/ dark cycle. $w^{1118 ; S x 1 a-m E o s 2 / C y o ; U A S-d T r p A 1 ~ t r a n s-~}$ genic lines were crossed with $\mathrm{w}^{1118} ;+/+; \mathrm{R} 57 \mathrm{C} 10-\mathrm{Gal} 4$ virgin females to generate the $\mathrm{w}^{1118}$;Sx1a-mEos2/+;UASdTrpA1/R57C10-Gal4 flies which were used throughout this study. Flies were raised at $19^{\circ} \mathrm{C}$ after which point females of the required genotype were collected under brief $\mathrm{CO}_{2}$ exposure and then kept at $19^{\circ} \mathrm{C}$ on a $12 / 12 \mathrm{~h}$ light/ dark cycle for 3-5d before experiments. Keeping the flies at $19^{\circ} \mathrm{C}$ prevented activation of $\mathrm{dTrpA} 1$ channels. The effectiveness of dTrpA1 was confirmed by exposing flies briefly to $30^{\circ} \mathrm{C}$, which rapidly induced paralysis (Movie 4).

\section{Imaging solution}

Modified hemolymph-like 3.1 (HL3.1) solution was prepared fresh on the day of an experiment and used both as a dissecting 


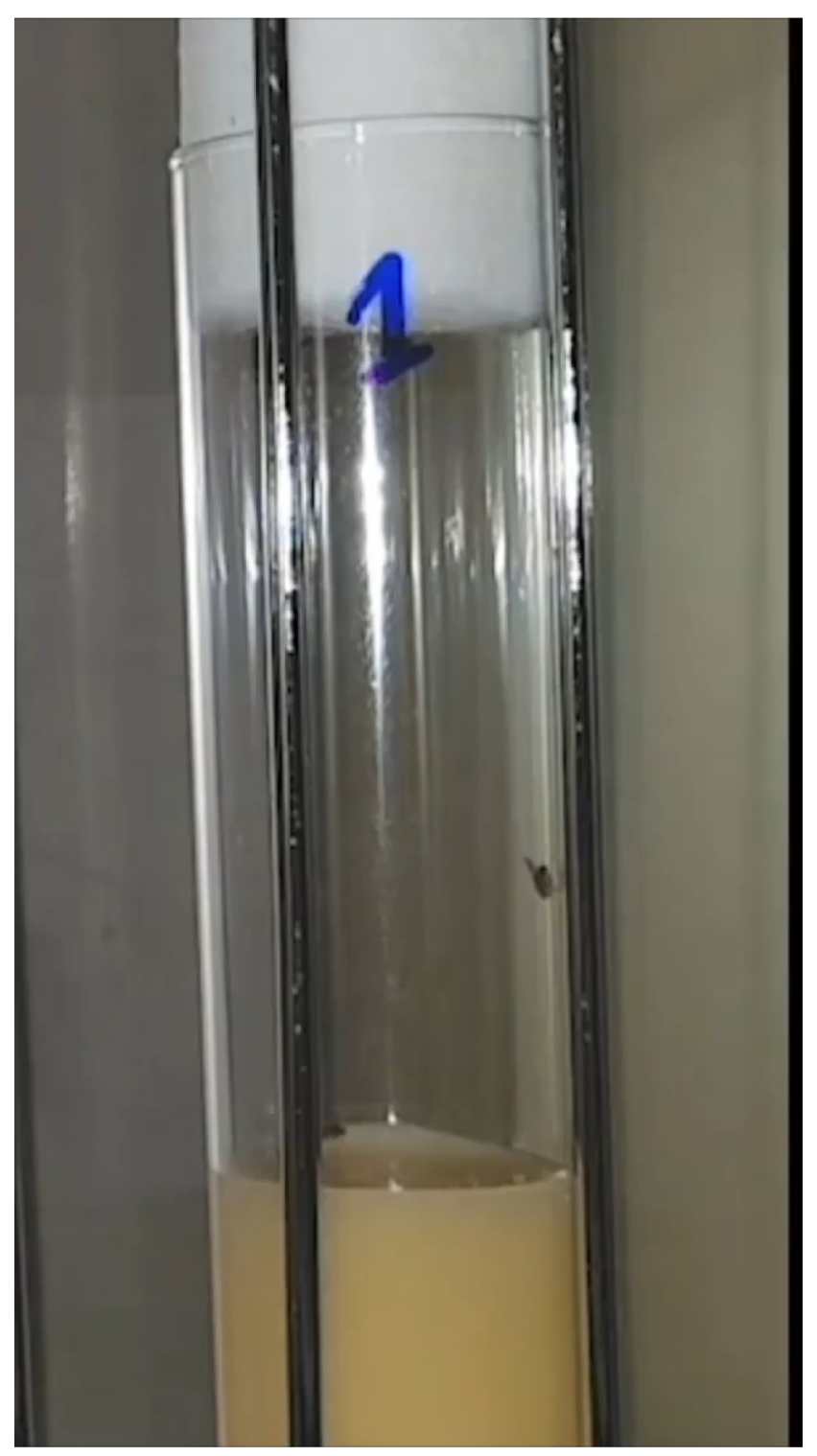

Movie 4. Conditional paralysis at $30^{\circ} \mathrm{C}$ in w1118;Sx1a-mEos2/ $+;$ UAS-dTrpA1/R57C10-Gal4 flies and lack of paralysis at $30^{\circ} \mathrm{C}$ in w1118;Sx1a-mEos2/+;UAS-dTrpA1/+ controls. [View online]

and imaging buffer. HL3.1 consists of $70 \mathrm{~mm} \mathrm{NaCl}, 5 \mathrm{mmKCl}, 1.5$ $\mathrm{mmCaCl}_{2}, 2 \mathrm{~mm} \mathrm{MgCl} 2,5 \mathrm{~mm}$ HEPES, $115 \mathrm{~mm}$ sucrose, $5 \mathrm{~mm}$ trehalose, and $\mathrm{pH} 7.2$ with $\mathrm{NaHCO}_{3}$ (Sigma-Aldrich).

Modified hemolymph-like 3 (HL3) solution used in Extended Data Figure 3-1 consisted of $70 \mathrm{~mm} \mathrm{NaCl}, 5$ mmKCl, $1.5 \mathrm{~mm} \mathrm{CaCl}_{2}, 20 \mathrm{~mm} \mathrm{MgCl}_{2}$, 5 mм HEPES, 115 $\mathrm{mm}$ sucrose, $5 \mathrm{~mm}$ trehalose, and $\mathrm{pH} 7.2$ with $\mathrm{NaHCO}_{3}$ (Sigma-Aldrich). Artificial CSF (aCSF) contained $25 \mathrm{~mm}$ HEPES, $120 \mathrm{~mm} \mathrm{NaCl}, 5 \mathrm{mmKCl}, 2 \mathrm{~mm} \mathrm{CaCl}_{2}, 2 \mathrm{~mm}$ $\mathrm{MgCl}_{2}$, and $30 \mathrm{~mm}$ glucose buffered to a $\mathrm{pH}$ of 7.4 using $\mathrm{NaOH}$.

\section{Anesthetics}

All anesthetic drugs were diluted into HL3.1 and mixed by vigorous vortexing for $\sim 1 \mathrm{~min}$. For intravenous anesthetics, except for ketamine, these were first diluted from stock in dimethyl sulfoxide (DMSO, Sigma-Aldrich D5879-500MI). Relevant concentrations were determined as previously described but not matched for equipotency (Zalucki et al., 2015; Bademosi et al., 2018b). Volatile anesthetics were taken directly from a stock bottle using a $10 \mu \mathrm{L}$ Hamilton syringe (Hamilton Company). A fresh preparation of HL3.1 solution with volatile anesthetics was made for each dissection. Estimates of isoflurane and sevoflurane concentrations were based on previous work (Sandstrom, 2004; Zalucki et al., 2015): 3 and $6 \mu \mathrm{l}$ of $100 \%$ stock of isoflurane and sevoflurane were each diluted into $20 \mathrm{~mL}$ of HL3.1 solution, which correspond to $\sim 0.19$ and $0.38 \mathrm{~mm}$, respectively, based on chromatography results from multiple HL3.1 samples (Zalucki et al., 2015; Bademosi et al., 2018b). The following anesthetics were used: 2,6-diisopropylphenol (propofol; Sigma-Aldrich D126608-100G), etomidate (Sigma-Aldrich, E6530-10MG), ketamine (llium Ketamil, Provet), isoflurane (Henry Schein, 1182097), and sevoflurane (Fluorochem, 28523-86-6)

\section{Dissection of Drosophila brains}

The brains of 3- to 5-d old female Drosophila flies were removed using a standard dissecting technique (Wu and Luo, 2006) on a Sylgard (Dow Corning) dish after brief anesthesia on a $\mathrm{CO}_{2}$ pad. Females were chosen to keep sexual dimorphisms consistent between experiments. Using Dumont \#5 forceps (Fine Science Tools, 11251-10), heads were removed from the body and placed in HL3.1 solution. The proboscis was then removed to gain access to the inside of the cuticle. Carefully tearing away at the cuticle until the brain is released, the brains were cleared of all tracheal tissue. Dissected brains were then mounted in $\sim 10 \mu \mathrm{l}$ of HL3.1 on a glass slide (Superfrost, ThermoFisher), and sealed shut using a 25$\mathrm{mm}$ square cover glass (Menzel-Gläser, ThermoFisher) rimmed with silicone vacuum grease (Dow Corning) with a paintbrush. For fixed brain imaging, brains were dissected as usual and then fixed in $4 \%$ paraformaldehyde (PFA) for 40 min and then washed in HL3.1 solution. Brains were then mounted in the same manner and imaged.

\section{Super-resolution and PALM}

All imaging was performed on a standard Zeiss ELYRA PS.1 microscope fitted with a Zeiss Plan-APOCHROMAT $100 \times 1.4 \mathrm{nA}$ oil immersion objective, a Zeiss FC12 definite focus, and an iXon EMCCD $512 \times 512$-pixel camera (Andor, Oxford Instruments). Mounted brains were inverted so that the oil-objective touches the coverslip and the region of interest (ROI) was navigated visually using bright-field illumination. Brains were imaged at a HILO sheet angle of $47.3^{\circ}$ to improve the signal-to-noise ratio, with a $1.6 \times$ lens magnification, in TIRF high power mode. A 570-620 + 750 filter cube was employed to further improve the signal. In order to simultaneously photoconvert native mEos2 and record photoconverted particles, two lasers with 405 and $561 \mathrm{~nm}$ wavelengths, respectively, were used to perform PALM. The laser powers used were 
$25 \%$ of the $561-\mathrm{nm}$ laser, with an average power at the specimen of $0.21 \mathrm{~mW}$; $405-\mathrm{nm}$ laser power varied with different recordings, from $0.001 \%$ to $0.01 \%$ with a power at the specimen of $0.1 \mu \mathrm{W}$. Because of a high amount of auto-photoconversion that occurs in the bright-field light from brain dissections, we first allowed the photoconverted particles to bleach for $\sim 1 \mathrm{~min}$ without the $405-\mathrm{nm}$ illumination to establish a baseline. Drift during imaging was evaluated per brain at this step by finding stable bright spots, which are likely auto-fluorescing protein aggregates of unknown providence. With the 561-nm illumination, an ROI was drawn around the spot, followed by $3 \mathrm{~min}$ of continuous recording to see whether the spot moved out of the ROI. Drift was also evaluated after imaging using a Pearson cross-correlation of the maximum zprojection of the $25^{\circ} \mathrm{C}$ and $30^{\circ} \mathrm{C}$ recordings (Extended Data Fig. 3-3). Details of the Pearson calculations are described in the Data and Statistical Analysis section. Brains that drifted were discarded. Drift can often occur because of the movement of recording solution toward the periphery of the coverslip, which can be overcome by sealing the coverslip edges with silicone grease, decreasing the size of the coverslip or increasing the amount of imaging solution. Zeiss Zen 2012 software was used to set the imaging parameters and capture the recordings.

For dTRPA1 activation experiments, a Zeiss incubation chamber, Heating Unit XL S, and TempModule S (Zeiss) was used to set, change, and monitor recording temperatures. An initial baseline recording at $25^{\circ} \mathrm{C}$ was taken for all experiments (unless noted otherwise) which was then increased to $30^{\circ} \mathrm{C}$ to stimulate neurotransmission and perform a second recording at the same location. The power of the ultraviolet (UV)-405-nm laser was adjusted throughout recordings to maintain the number of stochastically switched mEos2 molecules. A minimum of 16,000 frames were captured at $30-\mathrm{ms}$ frame rate with continuous exposure, the lowest exposure time achievable with the hardware used, to ensure at least 1000 Sx1a-mEos2 trajectories were recorded per experiment.

\section{Western blotting}

$20 \times \mathrm{w}^{1118}$;Sx1a-mEos2/+;UAS-TrpA1/R57C10-Gal4 female flies aged 3- to 5-d old were briefly anesthetized on a $\mathrm{CO}_{2}$ pad and sorted before transferring to a $15-\mathrm{ml}$ falcon tube on dry ice. Flies were vortexed for $15 \mathrm{~s}$ twice to separate the heads from the body. No. 25 and No. 40 standard sieves (Endecotts Ltd.) prechilled at $-80^{\circ} \mathrm{C}$ were used to separate heads from the body and legs. Heads were collected into a prechilled $1.5 \mathrm{ml}$ Eppendorf tube on dry ice with $30 \mu \mathrm{l}$ of a $1 \%$ Triton X-100 lysis buffer containing a 1:100 EDTA-free protease inhibitor cocktail. Heads were homogenized with a 1/4" ceramic sphere (MP, catalog \#6540-034) in a QIAGENTissueLyser LT. Homogenate was centrifuged for $20 \mathrm{~min}$ at $14,000 \mathrm{rpm}$ at $4^{\circ} \mathrm{C}$ to separate cellular debris from the lysate. The lysate was then added to $2 \times$ SDS loading buffer and boiled for $10 \mathrm{~min}$ at $100^{\circ} \mathrm{C} ; 30 \mu \mathrm{l}$ of the boiled sample was immediately loaded into a Mini-PROTEAN TGX 4-15\% gel (Bio-Rad catalog \#456-1083) and separated at $110 \mathrm{~V}$. Gel was then transferred onto an Immobilon-P membrane (Merck, catalog
\#IPVH00010) at $100 \mathrm{~V}$. The membrane was blocked in TBST (TBS $+1 \%$ Tween) solution containing $5 \%$ milk for $1 \mathrm{~h}$ at room temperature and washed $3 \times$ with TBS after which it was incubated overnight at $4^{\circ} \mathrm{C}$ with an anti-Sx1a antibody (Developmental Studies Hybridoma Bank, catalog \#8C3) diluted 1:1000 in a TBST solution with gentle agitation. The following morning, the membrane was washed $3 \times$ in a TBST solution containing $1 \%$ milk and incubated with a secondary antibody (goat to mouse IgG HRP, Abcam catalog \#ab205719) in a 1:10,000 dilution for $1 \mathrm{~h}$. Membranes were washed $3 \times$ in TBS and visualized in a Pierce ECL Western blotting substrate (ThermoScientific, catalog \#32106) using a Li-cor Odyssey Fc. Protein was quantified using Image Studio Lite (LI-COR Biosciences).

\section{Data and statistical analysis}

All data were analyzed using the free Fiji software TrackMate (Tinevez et al., 2017) adapted into a custom MATLAB GUI called single particle analysis (SPA; available from https://github.com/AdamDHines/sptPALMAnalysis) which incorporates mean squared displacement (MSD) and diffusion coefficient calculations, performed on a Lenovo ThinkPad with Windows 10. The analysis guide is available as Extended Data Document 1. Single Sx1a-mEos2 molecules were localized using a Laplacian of Gaussian (LoG) detection algorithm, median filtering, and subpixel localization with a manually determined threshold value for each recording:

$$
g(x, y, t)=\frac{1}{2 \pi t} e^{-\frac{x^{2}+y^{2}}{2 t}} .
$$

To track single molecules between frames, a linear assignment problem (LAP) algorithm (Jaqaman et al., 2008) was used to link particles by minimizing a cost matrix of distance between detected particles in a frame to every particle in the next frame. A minimum of 6 and a maximum of 1000 spots per track were included for analysis of the MSD, which measures the distance a particle travels from its initial position and is calculated by:

$$
\begin{gathered}
M S D(n \times \Delta t)=\sum_{i=1}^{N-n} \\
\frac{[x((i+n) \times \Delta t)-x(i \times \Delta t)]^{2}+[y((i+n) \times \Delta t)-y(i \times \Delta t)]^{2}}{N-n} .
\end{gathered}
$$

The diffusion coefficient, $D$, was calculated for each MSD curve with linear fits of the first four time points using the following:

$$
\operatorname{MSD}(\tau)=\alpha+4 D \tau .
$$

$N$ is the number of data points, the offset constant $\alpha$ includes the effects of localization error and finite camera exposure, $\Delta t$ is the time interval between each frame, with $x$ and $y$ being spatial coordinates for localizations in each image. Mobile-to-immobile ratios were calculated by 
summing the relative frequency of molecules with a $\log _{10}$ diffusion coefficient of more and less than -1.6 and dividing the mobile by the immobile fraction, which translates to be $0.021 \mu \mathrm{m}^{2} \mathrm{~s}^{-1}$ (Constals et al., 2015).

The point spread function (PSF) half width and localization precision of Sx1a-mEos2 molecules was determined from a single brain that was fixed in 4\% PFA for $45 \mathrm{~min}$ before imaging using Zeiss ZEN 2012 software (Extended Data Fig. 1-4).

For all experiments using thermogenetic stimulation, the peak MSD value for the baseline condition was used to normalize all values of the MSD (Watts et al., 2014) for both unstimulated and stimulated conditions, such that the peak MSD value for the unstimulated condition was set to 1 (Extended Data Fig. 3-2). Diffusion coefficients and mobile-to-immobile ratios were not able to be calculated with normalized MSD curves. Pearson correlations were used to determine levels of drift by comparing the maximum z-projection of the $25^{\circ} \mathrm{C}$ and $30^{\circ} \mathrm{C}$ recordings to calculate Pearson coefficients, performed in ImageJ using the colocalization threshold function (Extended Data Fig. $3-3)$. The peak mobility point $(0.30 \mathrm{~s})$ of the normalized $30^{\circ} \mathrm{C}$ data were subtracted by the peak point of the $25^{\circ} \mathrm{C}$ internal control to derive $\delta$ mobility (Extended Data Fig. 3-3). The $\delta$ mobilities were plotted against calculated Pearson coefficients to develop a linear regression and derive an $R^{2}$ value. The area under the curve (AUC) was measured for each normalized MSD curve using GraphPad Prism 8, with a baseline starting at $Y=0$, ignoring peaks that are $<10 \%$ of the distance from minimum to maximum $Y$, and defining that all peaks must go above baseline. To compare the mean of internally controlled AUC values a Wilcoxon matched signed-rank test was used with a significance threshold of $p=0.05$. To compare the means of the AUC of different conditions to controls, a Kolmogorov-Smirnov test with a significance threshold of $p=0.05$ was used. MSD presented is \pm SD and AUC data are $\pm 5-95$ th percentile. 95\% confidence intervals (Cls) were calculated around the mean.

\section{Data availability}

The datasets supporting the current study will be made available on a public database (eSpace, The University of Queensland) on publication: https://espace.library.uq.edu.au/.

\section{Code accessibility}

The code/software described in the paper is freely available online at https://github.com/AdamDHines/sptPALMAnalysis.

\section{Results and Discussion}

\section{Localizing and tracking the mobility of Sx1a in the adult fly brain}

We employed sptPALM to image and track individual Sx1a molecules in the ex vivo brains of adult Drosophila fruit flies (Fig. 1A; Extended Data Fig. 1-1). Ex vivo fly brains in buffer solutions remain viable and physiologically healthy for several hours (Gu and O'Dowd, 2006; Raccuglia et al., 2019), allowing us to apply this preparation to live-cell microscopy. Sx1a was tagged on the

extracellular $\mathrm{C}$ terminus with the photoconvertible fluorophore mEos2 (McKinney et al., 2009) and expressed pan-neuronally (Bademosi et al., 2016). Importantly, Sx1a-mEos2 expression was low relative to endogenous Sx1a in the adult fly brain (Extended Data Fig. 1-2), consistent with previous findings in larvae (Bademosi et al., 2016). Brains were mounted onto a glass slide in $\sim 10 \mu \mathrm{l}$ of fresh modified hemolymph-like solution 3.1 (HL3.1; Feng et al., 2004) and sealed with a square coverslip (MenzelGläser, ThermoFisher) rimmed with vacuum grease (Dow Corning; Fig. 1A, lower). Light compression reduced the thickness of the brain from $\sim 120$ to $40 \mu \mathrm{m}$, allowing for the imaging of tissue in a HILO configuration while retaining neural circuit architecture (Fig. 1C; Extended Data Fig. 1-3). Spinning disk confocal imaging confirmed mEos2 expression in brain neurons (Fig. 1D). When observing the brain at $100 \times$ magnification, the PSF overlap of the unconverted green form of mEos2 does not allow for the resolution of individual molecules or structures within the fly brain (Fig. 1E). Upon exposure to a low intensity UV $(405 \mathrm{~nm})$ photoconverting stimulus, stochastically switched red mEos2 molecules can be visualized sparsely (Fig. 1F). In order to confirm that we were imaging mEos2 molecules, we compared spot counts in brains that had no UV exposure and saw a significant increase in single molecule detection with photoconversion (Extended Data Fig. 1-4). At 30-ms exposure time, Sx1amEos2 molecules can be seen moving inside of neurons of the fly brain (Movie 1). We were able to achieve a localization precision of $\sim 18 \mathrm{~nm}$, which is close to previously reported measures (Extended Data Fig. 1-4; McKinney et al., 2009). Neural structures in the fly brain become evident after performing a maximum projection of a time series of PALM experiments (Fig. 1G), confirming that Sx1a-mEos2 is confined.

In order to characterize the mobility of individual tagged proteins, we performed SPT (Extended Data Fig. 1-1) as a post hoc step to image acquisition. We analyzed on average 2000-3000 individual trajectories of single Sx1amEos2 molecules over 16,000 frames (Fig. 1H) using the ImageJ software TrackMate (Tinevez et al., 2017) to localize molecules and perform particle tracking. Adapting TrackMate into a custom MATLAB interface, we analyzed the MSD (Fig. 1/) and molecule diffusion coefficients (Joensuu et al., 2017; Fig. 1J). On average, 10 molecules per frame were localized, with the majority of trajectories lasting 8 frames before terminating (Extended Data Fig. 14). We confirmed our analysis software by comparing our results with MSD data calculated using PALM-Tracer (a particle tracking plugin used in MetaMorph, Molecular Devices). Results were identical using either software (Extended Data Fig. 1-5).

To validate the reproducibility of our approach, we compared Sx1a-mEos2 mobility across successive recording sessions from the same brains. We recorded from different brain regions (Fig. $2 A-C$ ) and from the same brain region (Fig. $2 F-H$ ). We observed considerable variability in Sx1a-mEos2 mobility across experiments and 


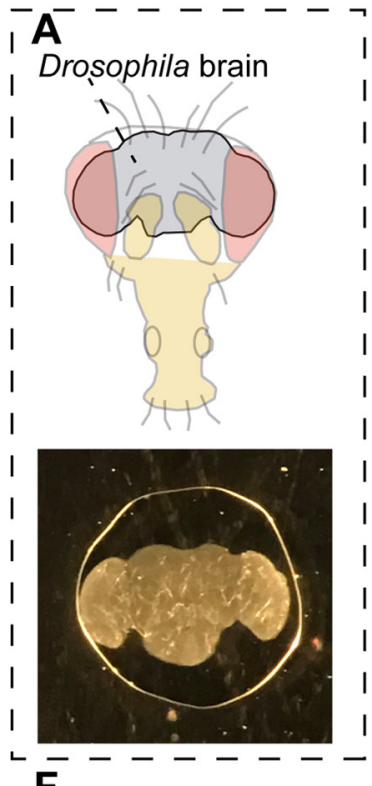

$\mathbf{F}$
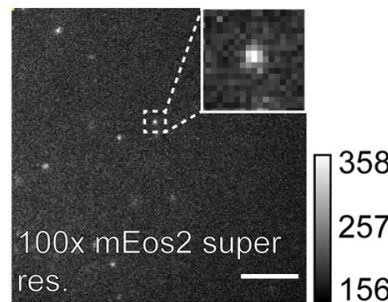

I

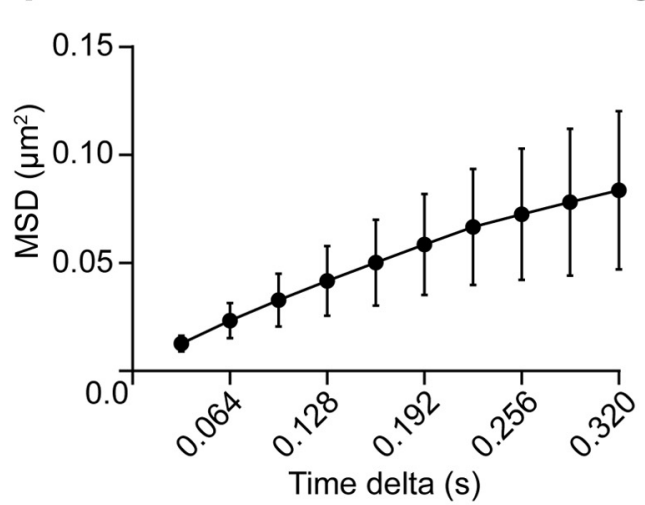

B

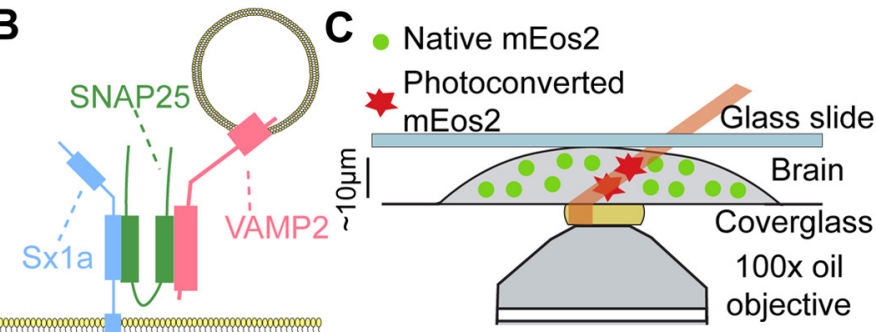

Highly inclined and

laminated optical sheet (HILO)

E

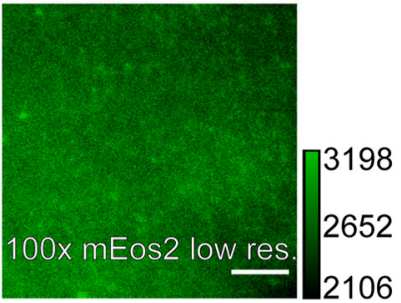

H

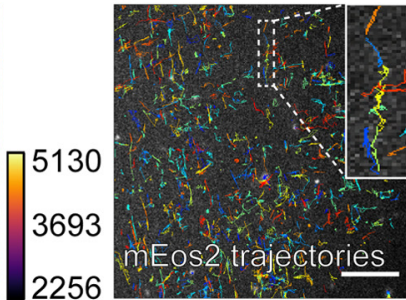

J

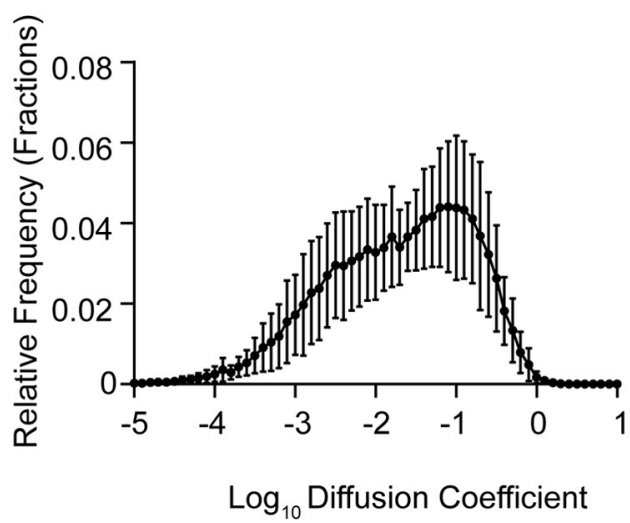

Figure 1. Imaging single Sx1a molecules in adult Drosophila brains. $\boldsymbol{A}$, The brain of adult Drosophila fruit flies (top) is dissected and mounted in a HL3.1 buffer and sealed between a glass slide and coverslip (bottom). B, Schema of the protein of interest being imaged, Sx1a (blue), with its SNARE partners SNAP25 (green), and VAMP2 (red). Sx1a is tagged with the photoconvertible fluorophore mEos2 on the $\mathrm{C}$ terminus. $\mathbf{C}$, Sx1a-mEos2-expressing brains are imaged under a HILO sheet illumination with simultaneous UV405-nm photoconverting and 561-nm recording lasers. $\boldsymbol{D}$, A $10 \times$ confocal image showing expression of mEos2 (native non-photoconverted green form) across the entire fly brain (scale bar: $100 \mu \mathrm{m}$, right calibration scale). $\boldsymbol{E}$, Green form of mEos2 expression at $100 \times$ magnification, individual molecules cannot be resolved because of PSF overlap (scale bar: $5 \mu \mathrm{m}$, right calibration scale). $\boldsymbol{F}$, Stochastically photoconverting mEos2 with a UV-405-nm laser can resolve single Sx1a-mEos2 molecules using a 561-nm laser without any PSF overlap (scale bar: $5 \mu \mathrm{m}$, right calibration scale). Inset, digital zoom of one molecule. G, Neuropil ultrastructure in the fly brain becomes apparent following a maximum intensity projection of all photoconverted mEos2 molecules over 16,000 frames of acquisition (scale bar: $5 \mu \mathrm{m}$, right calibration scale). Inset, digital zoom of one neuronal compartment. $\boldsymbol{H}, \mathrm{SPT}$ is performed on all detected Sx1a-mEos2 to track individual Sx1a molecules. Inset, Individual trajectories in different colors. I,J, Analysis of Sx1a-mEos2 trajectories reveals the mobility of Sx1a-mEos2 by calculating the MSD and diffusion coefficients of single trajectories $(n=13$ brains, data are \pm SD). See Extended Data Figures 1-1, 1-2, 1-3, 1-4, 1-5. 
A

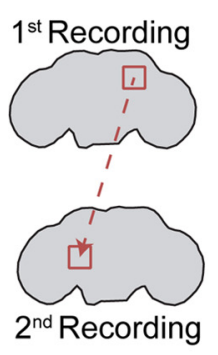

B

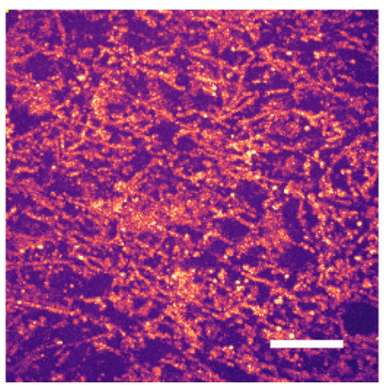

C

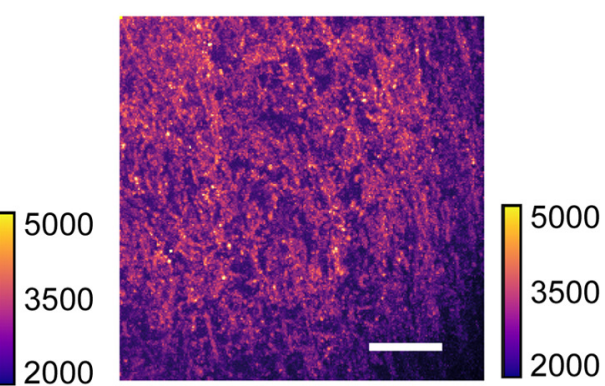

Brain

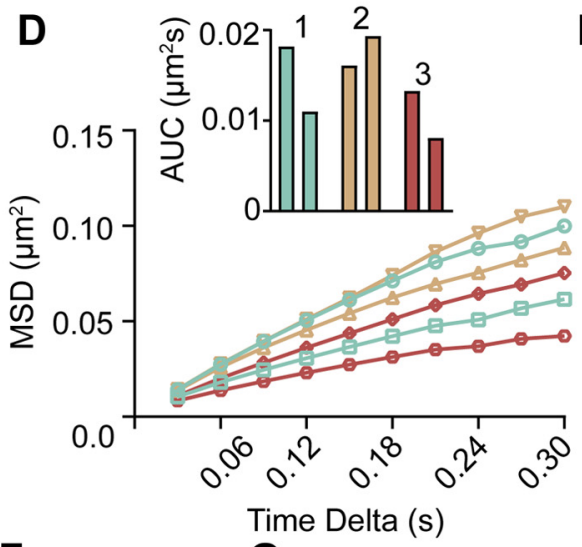

F

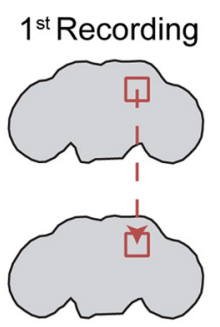

$2^{\text {nd }}$ Recording
G

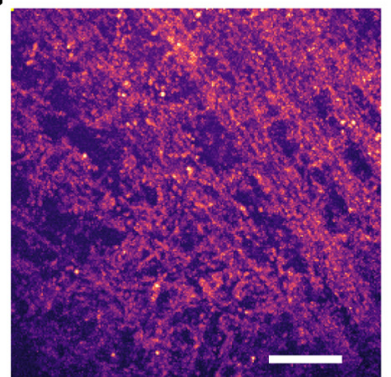

E

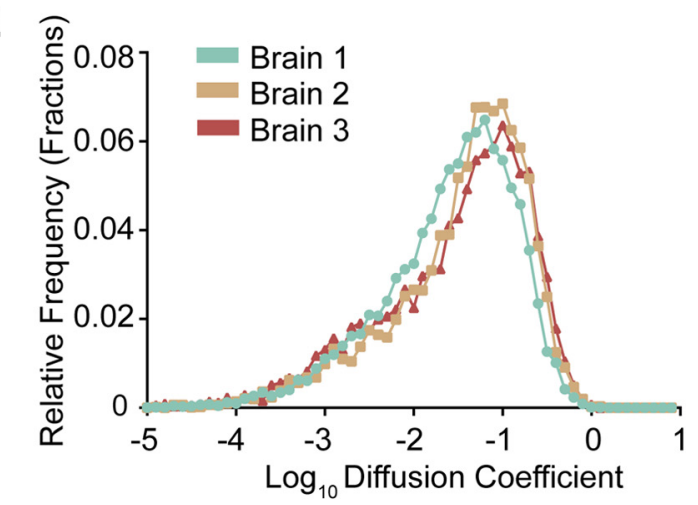

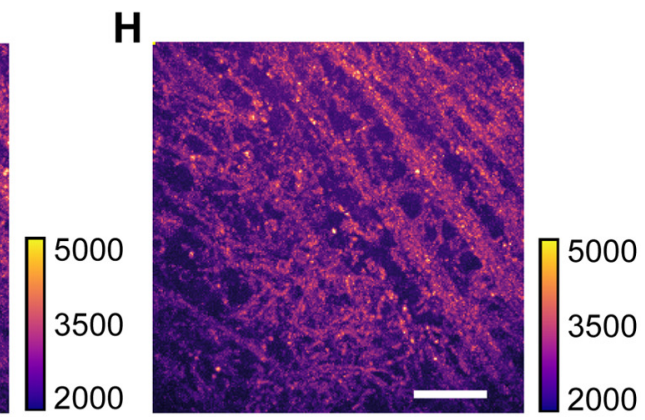
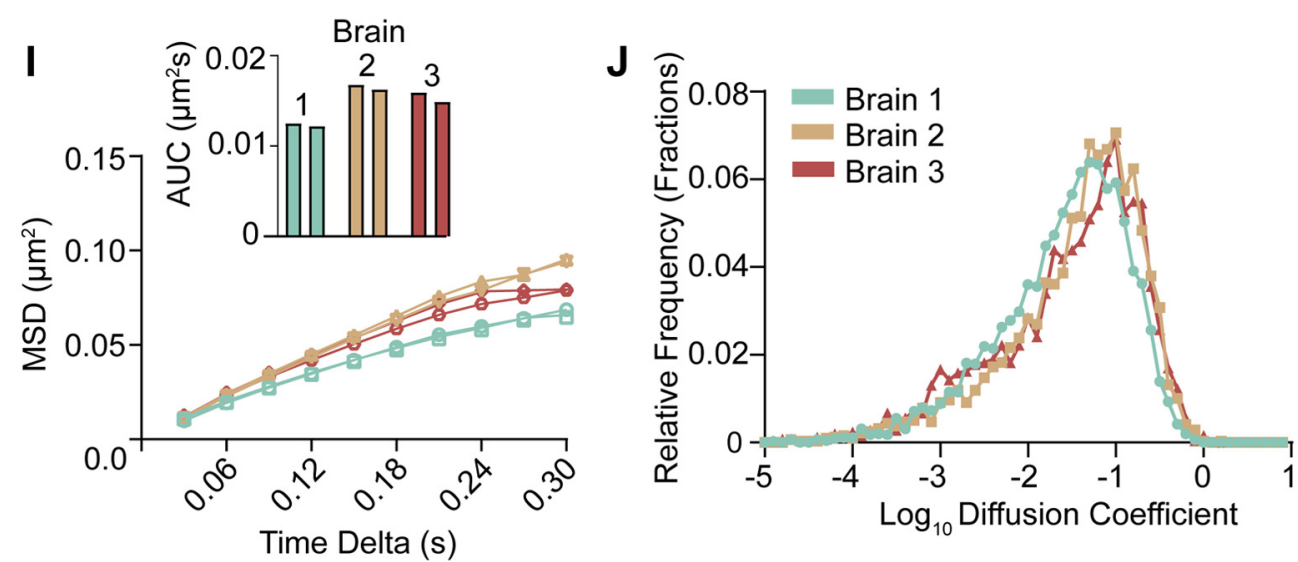

Figure 2. Tracking Sx1a-mEos2 mobility from the same brain region is highly reproducible. $\boldsymbol{A}$, Two recordings were taken from different regions in the same brain to establish whether differences in Sx1a-mEos2 mobility are observed. B,C, Maximum stack projections as in Figure $1 G$ for the two distinct brain regions as shown in $\boldsymbol{A}$ reveals different distribution of Sx1a-mEos2 molecules. $\boldsymbol{D}$, The MSD and AUC for two successive recordings in three separate brains highlights that within a brain there are different levels of Sx1amEos2 mobility, resulting in different diffusion coefficient estimates $(\boldsymbol{E})$ across experiments. $\boldsymbol{F}$, Two recordings were taken from the same brain region, to determine whether Sx1a-mEos2 mobility was consistent. $\mathbf{G}, \boldsymbol{H}$, Maximum stack projections for the same brain region recorded twice highlights that the neuronal structure remains the same. I, When Sx1a-mEos2 is tracked in the same region 
continued

twice, the MSD and AUC remain consistent, with similar diffusion coefficients $(\boldsymbol{J})$, providing a framework for internally controlled experiments performed at the same recording site. Scale bars: $5 \mu \mathrm{m}$, calibration scales to the right of each. See Extended Data Figure $2-1$.

brain regions (Fig. 2D,E), consistent with the large range in MSDs observed in our first dataset (Fig. 1/). Crucially, successive recordings from the same region (top right of the central brain, approximately in the lateral protocerebrum (Extended Data Fig. 1-3) revealed a high level of consistency in the number of localizations, trajectories, and MSD values within a recording site (Fig. 2/). This shows that results are repeatable in the same location, but also that some variability in diffusion coefficients exists across experiments in different brains (Fig. 2J). Importantly, successive recordings from the same brain region retained a similar number of localizations and trajectories, evident in highly comparable maximum projections of all the single molecule tracks (Fig. 2G,H) and the unchanged average spot and trajectory counts (Extended Data Fig. 2-1). We therefore proceeded with an internally controlled strategy centered on conditional neural activation in sequential recordings from the exact same location.

\section{Conditional activation of brain neurons increases Sx1a mobility}

Since the ionic composition of Drosophila extracellular fluid buffers varies in different experimental paradigms and can alter neuronal excitability (Feng et al., 2004), we examined the effects of different imaging solutions (Extended Data Fig. 3-1) and focused on HL3.1 buffer for all subsequent experiments. To ensure that the observed protein mobility was biologically relevant and not an artifact arising from the imaging solution, we performed the same experiment on brains that were first fixed in 4\% PFA and then imaged in HL3.1 solution. Fixing the tissue resulted in a complete loss of Sx1a-mEos2 mobility (Extended Data Fig. 3-1; Movie 2). In addition to this, imaging only HL3.1 solution without any brain tissue revealed highly mobile bright spots that could be localized, but not tracked using our SPA software (Movie 3).

We next investigated whether we could increase Sx1amEos2 mobility when we stimulated neurons. In previous work, we have shown that Sx1a-mEos2 mobility increases on stimulation of larval motor nerve terminals, most likely because of the recruitment of Sx1a molecules to sites of active zones to form SNARE complexes, and that sustained activation of dTrpA1 channels leads to a consistent increase in spontaneous miniature junction potential frequency (Bademosi et al., 2016). To stimulate neurons in the adult fly brain, we employed a temperature-sensitive Drosophila transient receptor potential cation channel 1a (dTrpA1; Fig. 3A), which we expressed under UAS control using the pan-neuronal driver R57C10-Gal4 (Jenett et al., 2012), thereby allowing co-expression with Sx1a-mEos2. Conditional activation of $\mathrm{dTrpA} 1$ at $30^{\circ} \mathrm{C}$ from a baseline of $25^{\circ}$ $\mathrm{C}$ allowed internally controlled experiments to be performed on the same recording site in the brain (Fig. 3B). Thus, all neuronal stimulation data could be normalized to the $25^{\circ} \mathrm{C}$ unstimulated condition at that recording site, thereby controlling for the variability observed across recording sites (Extended Data Fig. 3-2). To address potential drift in the tissue sample, we performed a cross-correlation analysis on the maximum projection data before and after dTrpA1 stimulation (Extended Data Fig. 33). We observed a consistent and significant increase in Sx1amEos2 mobility following thermogenetic stimulation, compared with baseline unstimulated conditions $(n=13, p=0.0002$, Wilcoxon test; Fig. $3 C, D$ ). In contrast, no significant increase in Sx1a-mEos2 mobility was observed at the elevated temperature in control brains that did not express dTrpA1 (Fig. 3E,F).

\section{General anesthetics restrict Sx1a mobility in brain neurons}

Having conditionally increased Sx1a-mEos2 mobility in the fly brain, we next sought to pharmacologically perturb this effect in the same preparation. We have previously shown that the intravenous general anesthetics propofol and etomidate decrease Sx1a-mEos2 mobility in mammalian neurosecretory cells as well as in Drosophila motor nerve terminals, by clustering Sx1a molecules on the presynaptic membrane (Bademosi et al., 2018b; Fig. 4A). Importantly, immobilization of Sx1a by propofol required a SNARE interaction domain; without this domain, propofol instead increased Sx1a mobility, as might be predicted because of increased membrane fluidity (Bahri et al., $2005,2007)$. We therefore next investigated whether intravenous general anesthetics also decreased Sx1amEos2 mobility in the adult Drosophila brain, employing our internally controlled strategy. Consistent with our previous findings in other systems (Bademosi et al., 2018b), we found that $3 \mu \mathrm{m}$ propofol and $8 \mu \mathrm{m}$ etomidate impaired Sx1a-mEos2 mobility in fly brain neurons (Fig. 4B,D). Also consistent with previous work in mammalian cells and fly larvae (Herring et al., 2011; Bademosi et al., 2018b), an analog of propofol failed to restrict Sx1a-mEos2 mobility in the adult fly brain (Extended Data Fig. 4-1). We then proceeded to test other general anesthetics, to see whether different categories of drugs also had this immobilizing effect on Sx1a. In contrast to propofol and etomidate, the NMDA-acting sedative ketamine $(100 \mu \mathrm{M})$ did not affect Sx1a-mEos2 mobility (Fig. 4C,D). We next tested two volatile drugs, isoflurane $(0.19 \mathrm{~mm})$ and sevoflurane $(0.38 \mathrm{~mm})$, and found that only isoflurane significantly impaired Sx1A-mEos2 mobility (Fig. 4C,D). We chose these concentrations approximating equipotency: the corresponding concentrations of isoflurane and sevoflurane in air ( $\sim 0.4 \%$ and $\sim 0.8 \%$, respectively) both achieve significant behavioral effects in fruit flies (Zalucki et al., 2015; Olufs et al., 2018). The effect of isoflurane on Sx1a mobility was large enough to be evident even without requiring normalization (see non-normalized isoflurane data compared with propofol in Extended Data Fig. 4-2). In the clinic, propofol and sevoflurane are often used sequentially to maintain general anesthesia during surgery (Harris et al., 2006). We therefore questioned 
A

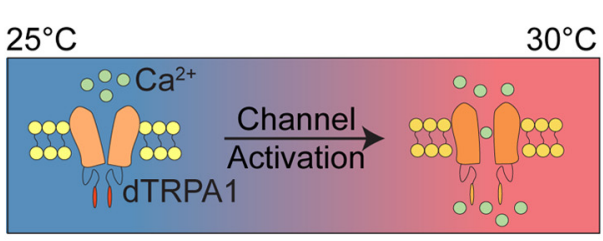

C dTrpA1 Expression

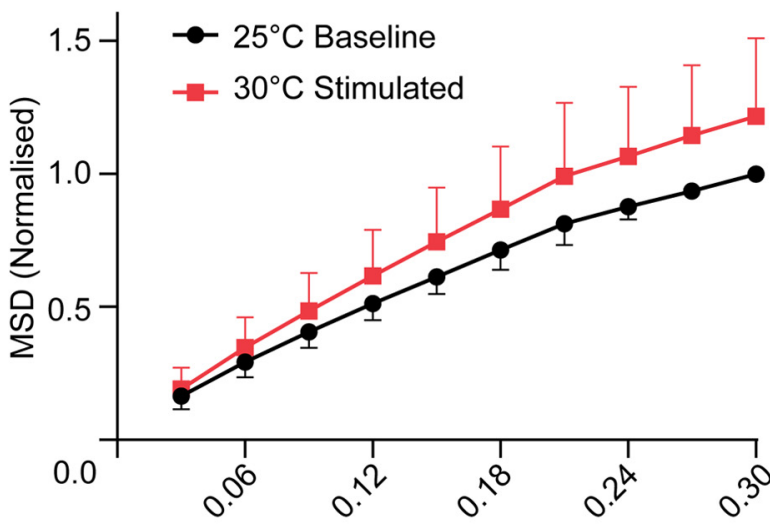

Time Delta (s)

\section{E}

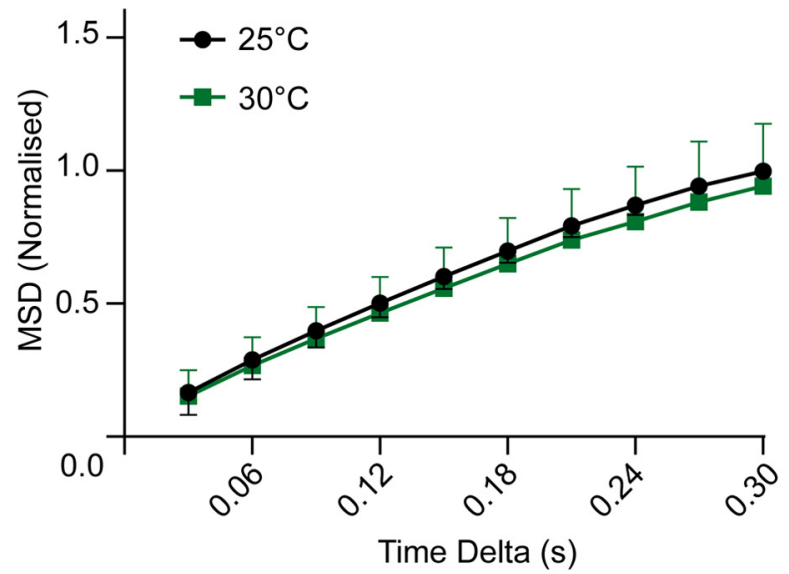

$1^{\text {st }}$ Recording

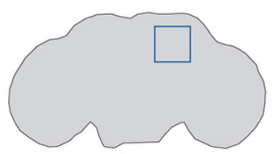

$3 \mathrm{~min}$

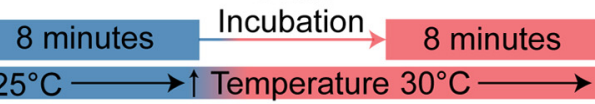

D
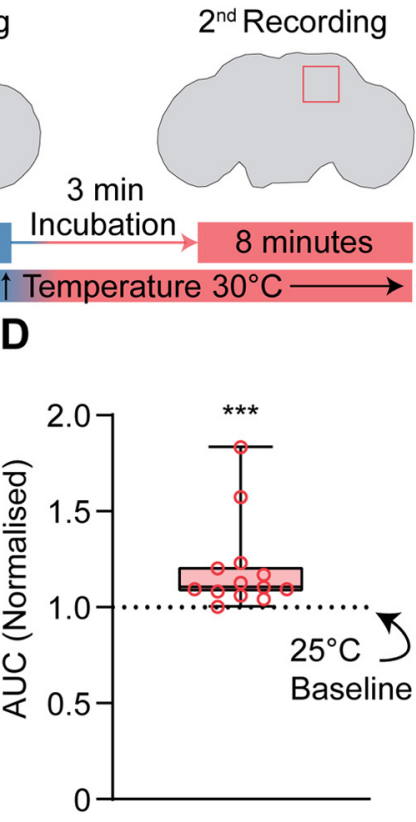

$\mathbf{F}$

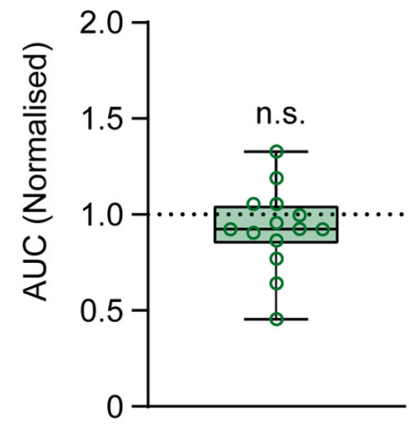

Figure 3. Neuronal stimulation increases the mobility of Sx1a-mEos2. A, Schematic of the Drosophila transient receptor potential cation channel type $\mathrm{A} 1$ (dTrpA1) function. At $25^{\circ} \mathrm{C}$ dTrpA1channels remain closed; increasing ambient temperature to $30^{\circ} \mathrm{C}$ activates these channels, resulting in $\mathrm{Ca}^{2+}$ influx and neuronal depolarization. $\boldsymbol{B}$, To measure the effects of dTrpA1 activation in the fly brain, recordings were taken from the same brain region twice: baseline recording was at $25^{\circ} \mathrm{C}$ followed by recording at $30^{\circ} \mathrm{C}$ after increasing incubation temperatures. $\boldsymbol{C}, \boldsymbol{D}, \mathrm{dTrpA} 1 \mathrm{stimulation}$ of fly neurons increased the mobility of Sx1a-mEos2 molecules compared with baseline (dotted line). All experiments were normalized to their own internal control at $25^{\circ} \mathrm{C}(n=13, p=0.0002$, Wilcoxon test; AUC, AUC 95\% Cl 1.059-1.347, data for MSD is \pm SD, data for AUC is $\pm 5-95$ th percentile). $\boldsymbol{E}, \boldsymbol{F}$, In the absence of the R57C10-Gal4 driver, no dTrpA1 was expressed in fly neurons and Sx1a-mEos2 mobility was not increased at $30^{\circ} \mathrm{C}$ $\left(n=14, p=0.1531\right.$, AUC $95 \% \mathrm{Cl} 30^{\circ} \mathrm{C} 0.8029-1.051$, Wilcoxon test, data for MSD is \pm SD, data for AUC is $\pm 5-95$ th percentile). See Extended Data Figures 3-1, 3-2, 3-30. n.s., not significant. ${ }^{\star \star} p<0.001$.

whether these intravenous and volatile drugs might have an additive effect on Sx1a mobility. Combining propofol with sevoflurane again significantly impaired Sx1a-mEos2 mobility, although not more so than propofol alone (Fig. $4 B, D)$. Taken together, our anesthesia results show that the adult fly brain provides a physiologically relevant platform to study the effect of different drugs on single- molecule behavior in intact neural tissue. We show that Sx1a is highly dynamic in the adult fly brain, with increased mobility following neural stimulation and decreased mobility under exposure to certain general anesthetics. This confirms and expands findings in other model systems (Bademosi et al., 2016, 2018b), and shows that some commonly used intravenous and volatile 
A

\section{Baseline}

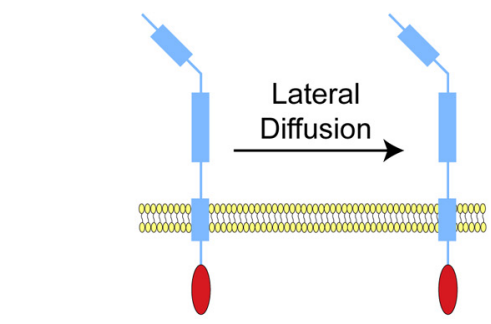

B

- HL3.1+DMSO - Propofol

\ Etomidate r Sevoflurane + Propofol

\section{General anesthetics}

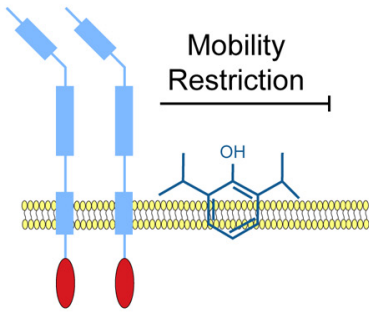

C

- HL3.1 - Isoflurane

^Sevoflurane Ketamine

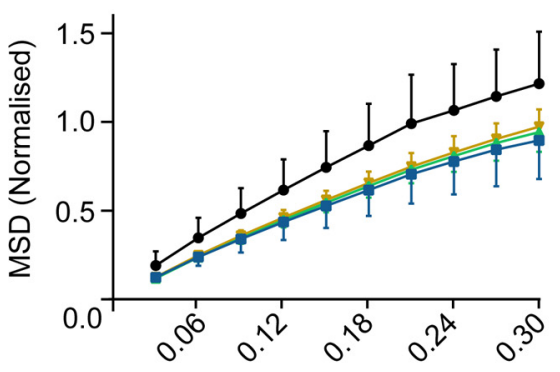

Time Delta (s)

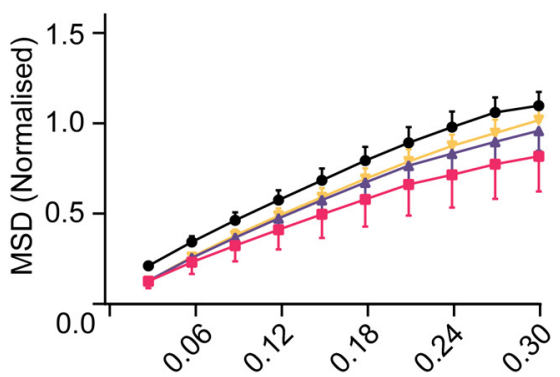

Time Delta (s)

D

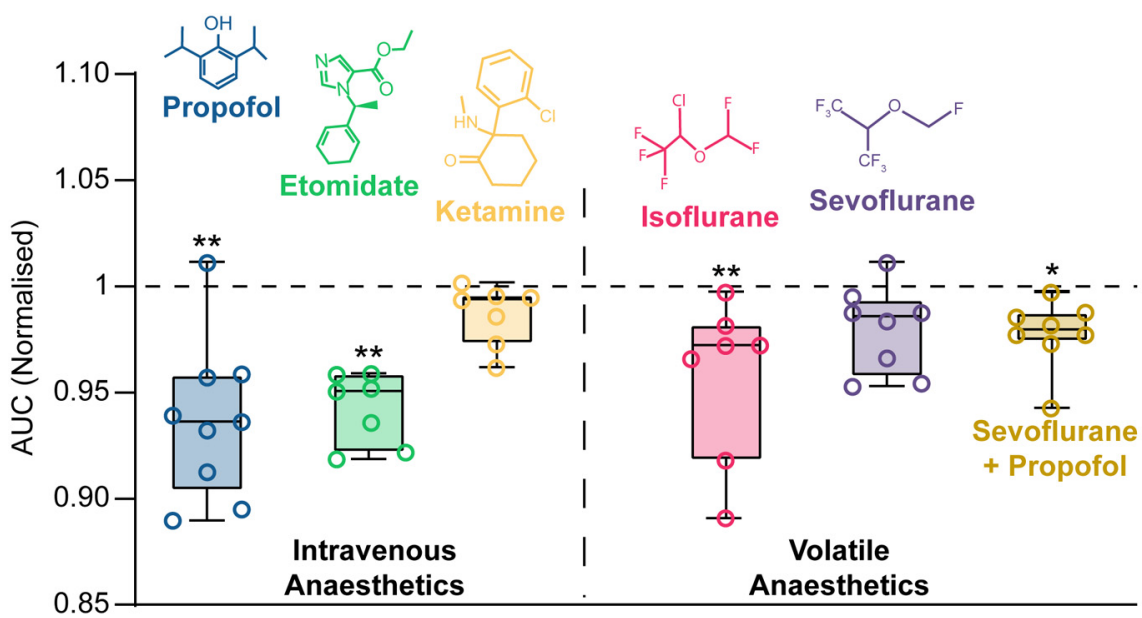

Figure 4. General anesthetics restrict Sx1a-mEos2 mobility in adult Drosophila brains. $\boldsymbol{A}$, left,Sx1a-mEos2 is able to diffuse laterally across a membrane, but mobility becomes restricted in the presence of propofol (right). $\boldsymbol{B}$, Normalized MSD curves comparing all anesthetics under stimulation that contained DMSO in the HL3.1. $\boldsymbol{C}$, Same as $\boldsymbol{B}$ but without DMSO in the solution (MSD is normalized). All data are represented as \pm SD. $\boldsymbol{D}$, Intravenous and volatile general anesthetics restrict the mobility of Sx1a-mEos2 compared with respective controls (dashed line). Both propofol $(3 \mu \mathrm{M})$ and etomidate $(8 \mu \mathrm{M})$ significantly reduced Sx1A-mEos 2 mobility (AUC) when compared with a HL3.1+DMSO control (propofol $n=9, p=0.0009$, AUC 95\% Cl 0.908-0.966; etomidate $n=8$, $p=0.0055$, AUC $95 \% \mathrm{Cl} 0.927-0.958$, Kruskal-Wallis test, data are $\pm 5-95$ th percentile). Ketamine (100 $\mu \mathrm{m})$ was unable to restrict Sx1a-mEos2 mobility when compared with a HL3.1 control $(n=6, p=0.9924$, AUC 95\% Cl 0.974-1.00, Kruskal-Wallis test, data are $\pm 5-95$ th percentile). The volatile anesthetic isoflurane $(0.19 \mathrm{~mm})$ was able to restrict Sx1a-mEos2 mobility but sevoflurane $(0.38$ $\mathrm{mM}$ ) was not, compared with a HL3.1 control (isoflurane $n=9, p=0.0079$, AUC 95\% Cl 0.922-0.992; sevoflurane $n=8, p=0.2672$, AUC $95 \% \mathrm{Cl}$ 0.963-0.998, Kruskal-Wallis test, data are $\pm 5-95$ th percentile). The addition of propofol (3 $\mu \mathrm{M})$ to sevoflurane significantly restricted Sx1a-mEos2 mobility compared with a HL3.1+DMSO control $(n=8, p=0.0108$, AUC 95\% Cl 0.965-0.992). See Extended Data Figures $4-1,4-2 .{ }^{*} p<0.05 ;{ }^{\star *} p<0.01$. 
general anesthetics might be affecting Sx1a mobility in the same manner. Importantly, we show the same effect for volatile as well as intravenous anesthetics, and that isoflurane in particular seems to have the greatest impact on Sx1a mobility.

In conclusion, we have shown that single mEos-tagged molecules can be resolved and tracked in the ex vivo brains of adult Drosophila fruit flies. This provides a useful and versatile tool for Drosophila researchers and those looking to perform super-resolution imaging of intact tissue, expanding on earlier inroads in this direction (Chen et al., 2014; Mir et al., 2018; Reisser et al., 2018). By employing an internally controlled paradigm, we were able to reliably increase the mobility of a presynaptic protein, Sx1a, through thermogenetic stimulation and restrict this mobility with the use of common general anesthetics. One caveat of our anesthetic results is that final concentrations in the brain tissue were approximated, based on previous experiments in other preparations. It remains possible, for example, that higher concentrations of sevoflurane or ketamine might also impair Sx1a mobility. Nevertheless, tracking single molecule dynamics in the ex vivo brains of adult Drosophila flies opens a new window into understanding the behavior of individual molecules in intact tissue, to, for example, help determine which mechanisms are drug-specific and which might reflect a common property of diverse drugs. Our results indicate that general anesthetics such as propofol and isoflurane might have similar effects among different kinds of chemical synapses. Although the adult fly brain is mostly cholinergic (Yasuyama and Salvaterra, 1999), we most likely sampled a variety of synapse types, including inhibitory synapses. That we found the same basic result (decreased Sx1a mobility) as in purely glutamatergic larval neuromuscular synapses (Bademosi et al., 2018b) argues for a common mechanism. Although our results focus on a ubiquitous presynaptic protein expressed in all neurons, the capacity to address circuit-specific questions could be expanded by adapting this approach to promoterdriven expression systems such as UAS/Gal4 on any protein target of interest. We believe this will result in highly reproducible and less variable results, as evidenced by the robustness of Sx1a-mEos2 mobility when recording in the same location twice. It will be interesting to apply SPT to investigate, for example, if $\mathrm{Sx} 1 \mathrm{a}$ is equally compromised at excitatory versus inhibitory synapses, or to examine the individual dynamics of other proteins under general anesthesia, such as receptors in dedicated sleep/wake circuits in the fly brain (Kottler et al., 2013; van Swinderen and Kottler, 2014). Finally, a major advantage of conducting this work in animal models such as Drosophila is the capacity to efficiently test behavioral relevance, for example, as a way to relate local effects at the synapse with higher order behavioral readouts in behaving animals (Zalucki et al., 2015; Troup et al., 2019; van Swinderen and Hines, 2020).

\section{References}

Axelrod D (2001) Total internal reflection fluorescence microscopy in cell biology.Traffic 2:764-774.

Bademosi AT, Lauwers E, Padmanabhan P, Odierna L, Chai YJ, Papadopulos A, Goodhill GJ, Verstreken P, van Swinderen B, Meunier FA (2016) In vivo single-molecule imaging of syntaxin1A reveals polyphosphoinositide- and activity-dependent trapping in presynaptic nanoclusters. Nat Commun 7:13660.

Bademosi AT, Lauwers E, Amor R, Verstreken P, van Swinderen B, Meunier FA (2018a) In vivo single-molecule tracking at the Drosophila presynaptic motor nerve terminal. J Vis Exp. Advance online publication. Retrived Jan 14, 2018. doi: 10.3791/56952.

Bademosi AT, Steeves J, Karunanithi S, Zalucki OH, Gormal RS, Liu S, Lauwers E, Verstreken P, Anggono V, Meunier FA, van Swinderen B (2018b) Trapping of syntaxin1a in presynaptic nanoclusters by a clinically relevant general anesthetic. Cell Rep 22:427-440.

Bahri MA, Heyne BJ, Hans P, Seret AE, Mouithys-Mickalad AA, Hoebeke MD (2005) Quantification of lipid bilayer effective microviscosity and fluidity effect induced by propofol. Biophys Chem 114:53-61.

Bahri MA, Seret A, Hans P, Piette J, Deby-Dupont G, Hoebeke M (2007) Does propofol alter membrane fluidity at clinically relevant concentrations? An ESR spin label study. Biophys Chem 129:82-91.

Baumgart JP, Zhou Z-Y, Hara M, Cook DC, Hoppa MB, Ryan TA, Hemmings HC (2015) Isoflurane inhibits synaptic vesicle exocytosis through reduced $\mathrm{Ca} 2+$ influx, not $\mathrm{Ca} 2+$-exocytosis coupling. Proc Natl Acad Sci USA 112:11959-11964.

Bennett M, Calakos N, Scheller R (1992) Syntaxin: a synaptic protein implicated in docking of synaptic vesicles at presynaptic active zones. Science 257:255-259.

Betzig E, Patterson GH, Sougrat R, Lindwasser OW, Olenych S, Bonifacino JS, Davidson MW, Lippincott-Schwartz J, Hess HF (2006) Imaging intracellular fluorescent proteins at nanometer resolution. Science 313:1642-1645.

Chen BC, Legant WR, Wang K, Shao L, Milkie DE, Davidson MW, Janetopoulos C, Wu XS, Hammer JA, Liu Z, English BP, MimoriKiyosue Y, Romero DP, Ritter AT, Lippincott-Schwartz J, FritzLaylin L, Mullins RD, Mitchell DM, Bembenek JN, Reymann AC, et al.(2014) Lattice light-sheet microscopy: imaging molecules to embryos at high spatiotemporal resolution. Science 346:1257998.

Constals A, Penn AC, Compans B, Toulmé E, Phillipat A, Marais S, Retailleau N, Hafner AS, Coussen F, Hosy E, Choquet D (2015) Glutamate-induced AMPA receptor desensitization increases their mobility and modulates short-term plasticity through unbinding from Stargazin. Neuron 85:787-803.

Couteaux R, Pecot-Dechavassine M (1970) [Synaptic vesicles and pouches at the level of 'active zones' of the neuromuscular junction.]C R AcadHebd Seances Acad Sci D 271:2346-2349.

Feng Y, Ueda A, Wu C (2004) A modified minimal hemolymph-like solution, hl3.1, for physiological recordings at the neuromuscular junctions of normal and mutant Drosophila larvae. J Neurogenet 18:377-402.

Fergestad T, Wu MN, Schulze KL, Lloyd TE, Bellen HJ, Broadie K (2001) Targeted mutations in the syntaxin H3 domain specifically disrupt SNARE complex function in synaptic transmission. J Neurosci 21:9142-9150.

Ferro-Novick S, Jahn R (1994) Vesicle fusion from yeast to man. Nature 370:191-193.

Fujiwara T, Mishima T, Kofuji T, Chiba T, Tanaka K, Yamamoto A, Akagawa $K$ (2006) Analysis of knock-out mice to determine the role of HPC-1/syntaxin $1 \mathrm{~A}$ in expressing synaptic plasticity. J Neurosci 26:5767-5776.

Gu H, O'Dowd DK (2006) Cholinergic synaptic transmission in adult Drosophila Kenyon cells in situ. J Neurosci 26:265-272.

Harris RS, Lazar O, Johansen JW, Sebel PS (2006) Interaction of propofol and sevoflurane on loss of consciousness and movement to skin incision during general anesthesia. Anesthesiology 104:1170-1175.

Hemmings HC, Yan W, Westphalen RI, Ryan TA (2005) The general anesthetic isoflurane depresses synaptic vesicle exocytosis. Mol Pharmacol 67:1591-1599.

Hemmings HC, Riegelhaupt PM, Kelz MB, Solt K, Eckenhoff RG, Orser BA, Goldstein PA (2019) Towards a comprehensive understanding of anesthetic mechanisms of action: a decade of discovery. Trends Pharmacol Sci 40:464-481.

Herring BE, McMillan K, Pike CM, Marks J, Fox AP, Xie Z (2011) Etomidate and propofol inhibit the neurotransmitter release machinery at different sites. J Physiol 589:1103-1115. 
Humphrey JA, Hamming KS, Thacker CM, Scott RL, Sedensky MM, Snutch TP, Morgan PG, Nash HA (2007) A putative cation channel and its novel regulator: cross-species conservation of effects on general anesthesia. Curr Biol 17:624-629.

Jaqaman K, Loerke D, Mettlen M, Kuwata H, Grinstein S, Schmid SL, Danuser G (2008) Robust single-particle tracking in live-cell timelapse sequences. Nat Methods 5:695-702.

Jenett A, Rubin GM, Ngo TTB, Shepherd D, Murphy C, Dionne H, Pfeiffer BD, Cavallaro A, Hall D, Jeter J, lyer N, Fetter D, Hausenfluck JH, Peng H, Trautman ET, Svirskas RR, Myers EW, Iwinski ZR, Aso Y, DePasquale GM, et al. (2012) A GAL4-driver line resource for Drosophila neurobiology. Cell Rep 2:991-1001.

Joensuu M, Martínez-Mármol R, Padmanabhan P, Glass NR, Durisic N, Pelekanos M, Mollazade M, Balistreri G, Amor R, Cooper-White JJ, Goodhill GJ, Meunier FA (2017) Visualizing endocytic recycling and trafficking in live neurons by subdiffractional tracking of internalized molecules. Nat Protoc 12:2590-2622.

Jung JH, Szule JA, Stouder K, Marshall RM, McMahan UJ (2018) Active zone material-directed orientation, docking, and fusion of dense core vesicles alongside synaptic vesicles at neuromuscular junctions. Front Neuroanat 12:72.

Karunanithi S, Cylinder D, Ertekin D, Zalucki OH, Marin L, Lavidis NA, Atwood HL, van Swinderen B (2020) Proportional downscaling ofglutamatergic release sites by the general anesthetic propofol at Drosophila motor nerve terminals. eneuro 7:ENEURO.0422-19.2020.

Kasula R, Chai YJ, Bademosi AT, Harper CB, Gormal RS, Morrow IC, Hosy E, Collins BM, Choquet D, Papadopulos A, Meunier FA (2016) The Munc18-1 domain 3a hinge-loop controls syntaxin-1A nanodomain assembly and engagement with the SNARE complex during secretory vesicle priming. J Cell Biol 214:847-858.

Kofuji T, Hayashi Y, Fujiwara T, Sanada M, Tamaru M, Akagawa K (2017) A part of patients with autism spectrum disorder has haploidy of HPC$1 /$ syntaxin1A gene that possibly causes behavioral disturbance as in experimentally gene ablated mice. Neurosci Lett 644:5-9.

Kottler B, Bao H, Zalucki O, Imlach W, Troup M, van Alphen B, Paulk A, Zhang B, van Swinderen B (2013) A sleep/wake circuit controls isoflurane sensitivity in Drosophila. Curr Biol 23:594-598.

Manley S, Gillette JM, Patterson GH, Shroff H, Hess HF, Betzig E, Lippincott-Schwartz J (2008) High-density mapping of single-molecule trajectories with photoactivated localization microscopy. Nat Methods 5:155-157.

Manzo C, Garcia-Parajo MF (2015) A review of progress in single particle tracking: from methods to biophysical insights. Rep Prog Phys 78:124601.

McKinney SA, Murphy CS, Hazelwood KL, Davidson MW, Looger LL (2009) A bright and photostable photoconvertible fluorescent protein. Nat Methods 6:131-133.

Mir M, Stadler MR, Ortiz SA, Hannon CE, Harrison MM, Darzacq X, Eisen MB (2018) Dynamic multifactor hubs interact transiently with sites of active transcription in Drosophila embryos. Elife 7:e40497.

Olufs ZPG, Loewen CA, Ganetzky B, Wassarman DA, Perouansky M (2018) Genetic variability affects absolute and relative potencies and kinetics of the anesthetics isoflurane and sevoflurane in Drosophila melanogaster. Sci Rep 8:2348.

Raccuglia D, Huang S, Ender A, Heim M-M, Laber D, Suárez-Grimalt R, Liotta A, Sigrist SJ, Geiger JRP, Owald D (2019) Network-specific synchronization of electrical slow-wave oscillations regulates sleep drive in Drosophila. Curr Biol 29:3611-3621.e3.

Reddy-Alla S, Böhme MA, Reynolds E, Beis C, Grasskamp AT, Mampell MM, Maglione $M$, Jusyte $M$, Rey $U$, Babikir $H$, McCarthy AW, Quentin C, Matkovic T, Bergeron DD, Mushtaq Z, Göttfert F, Owald D, Mielke T, Hell SW, Sigrist SJ, et al.(2017) Stable Positioning of Unc13 Restricts Synaptic Vesicle Fusion to Defined Release Sites to Promote Synchronous Neurotransmission. Neuron 95:1350-1364.e12.
Reisser M, Palmer A, Popp AP, Jahn C, Weidinger G, Gebhardt JCM (2018) Single-molecule imaging correlates decreasing nuclear volume with increasing TF-chromatin associations during zebrafish development. Nat Commun 9:5218.

Saifee O, Wei L, Nonet ML (1998) The Caenorhabditis elegans unc64 locus encodes a syntaxin that interacts genetically with synaptobrevin. Mol Biol Cell 9:1235-1252.

Sandstrom DJ (2004) Isoflurane depresses glutamate release by reducing neuronal excitability at the Drosophila neuromuscular junction. J Physiol 558:489-502.

Schulze KL, Broadie K, Perin MS, Bellen HJ (1995) Genetic and electrophysiological studies of Drosophila syntaxin-1A demonstrate its role in nonneuronal secretion and neurotransmission. Cell 80:311-320.

Stevens DR, Schirra C, Becherer U, Rettig J (2011) Vesicle pools: lessons from adrenal chromaffin cells. Front Synaptic Neurosci 3:2

Südhof TC (2012) The presynaptic active zone. Neuron 75:11-25.

Südhof TC, Rizo J (2011) Synaptic vesicle exocytosis. Cold Spring HarbPerspect Biol 3:a005637.

Tinevez JY, Perry N, Schindelin J, Hoopes GM, Reynolds GD, Laplantine E, Bednarek SY, Shorte SL, Eliceiri KW (2017) TrackMate: an open and extensible platform for single-particle tracking. Methods 115:80-90.

Tokunaga M, Imamoto N, Sakata-Sogawa K (2008) Highly inclined thin illumination enables clear single-molecule imaging in cells. Nat Methods 5:159-161.

Tønnesen J, Inavalli WGK, Nägerl UV (2018) Super-resolution imaging of the extracellular space in living brain tissue. Cell 172:1108-1121.e15.

Troup M, Zalucki OH, Kottler BD, Karunanithi S, Anggono V, van Swinderen B (2019) Syntaxin1A neomorphic mutations promote rapid recovery from isoflurane anesthesia in Drosophila melanogaster. Anesthesiology 131:555-568.

Ullrich A, Böhme MA, Schöneberg J, Depner H, Sigrist SJ, Noé F (2015) Dynamical organization of syntaxin-1A at the presynaptic active zone. PLoSComput Biol 11:e1004407.

van Swinderen B, Kottler B (2014) Explaining general anesthesia: a two-step hypothesis linking sleep circuits and the synaptic release machinery. Bioessays 36:372-381.

van Swinderen B, Hines AD (2020) Turning to Drosophila for help in resolving general anesthesia. Proc Natl Acad Sci USA 117:24627-24628.

van Swinderen B, Saifee O, Shebester L, Roberson R, Nonet ML, Crowder CM (1999) A neomorphicsyntaxin mutation blocks volatile-anesthetic action in Caenorhabditis elegans. Proc Natl Acad Sci USA 96:2479-2484.

Vardar G, Chang S, Arancillo M, Wu Y-J, Trimbuch T, Rosenmund C (2016) Distinct functions of syntaxin-1 in neuronal maintenance, synaptic vesicle docking, and fusion in mouse neurons. J Neurosci 36:7911-7924.

Watts F, Tan LE, Wilson CG, Girkin JM, Tassieri M, Wright AJ (2014) Investigating the micro-rheology of the vitreous humor using an optically trapped local probe. J Opt 16:015301.

Willig KI, Rizzoli SO, Westphal V, Jahn R, Hell SW (2006) STED microscopy reveals that synaptotagmin remains clustered after synaptic vesicle exocytosis. Nature 440:935-939.

Wu JS, Luo L (2006) A protocol for dissecting Drosophila melanogaster brains for live imaging or immunostaining. Nat Protoc 1:2110-2115.

Yasuyama K, Salvaterra PM (1999) Localization of choline acetyltransferase-expressing neurons in Drosophila nervous system. Microsc Res Tech 45:65-79.

Zalucki OH, Menon H, Kottler B, Faville R, Day R, Bademosi AT, Lavidis N, Karunanithi S, van Swinderen B (2015) Syntaxin1Amediated resistance and hypersensitivity to isoflurane in Drosophila melanogaster. Anesthesiology 122:1060-1074.

Zhang M, Chang H, Zhang Y, Yu J, Wu L, Ji W, Chen J, Liu B, Lu J, Liu Y, Zhang J, Xu P, Xu T (2012) Rational design of true monomeric and bright photoactivatable fluorescent proteins. Nat Methods 9:727-729. 